SHEP 97-06

April 1997

\title{
Derivative expansion of the renormalization group in $\mathrm{O}(\mathrm{N})$ scalar field theory
}

\author{
Tim R. Morris \\ and \\ Michael D. Turner \\ Physics Department, University of Southampton \\ Highfield, Southampton SO17 1BJ, U.K.
}

\begin{abstract}
We apply a derivative expansion to the Legendre effective action flow equations of $O(N)$ symmetric scalar field theory, making no other approximation. We calculate the critical exponents $\eta, \nu$, and $\omega$ at the both the leading and second order of the expansion, associated to the three dimensional Wilson-Fisher fixed points, at various values of $N$. In addition, we show how the derivative expansion reproduces exactly known results, at special values $N=\infty,-2,-4, \cdots$.
\end{abstract}




\section{Introduction}

In contrast to other analytic approaches, many different non-perturbative approximations can be envisaged within the Wilsonian renormalization group framework [1] which preserve the structure of the continuum limit (viz. renormalisability) [2]. This fact alone makes a Wilsonian renormalization group calculational framework potentially extremely powerful, and is surely in large part responsible for the resurgence of interest in these methods over the last few years. This framework holds other advantages also, for example enabling systematic searches for all (non-perturbative) continuum limits with the given field content and symmetries, and direct calculation of continuum quantities [2]-[5] (i.e. without introducing corresponding bare quantities). Against this background, it is surely important to test and understand as thoroughly as possible the reliability and accuracy of the different possible systematic non-perturbative approximation schemes.

Two such schemes appear particularly natural for the Wilsonian effective action: truncations in the operator content to a finite set of operators; or -less severely- an expansion and truncation in powers of space-time derivatives where no other approximation is made (a.k.a. "derivative expansion"). While carefully chosen truncations to a finite set of operators can be very successful in particular cases [6, 7, 8], there are reasons to expect these to have limited reliability and accuracy in general non-perturbative situations 制. It is also difficult to see how to keep such truncations systematic in practice beyond the lowest orders of the powers of derivatives they incorporate. In contrast, there are several reasons why one should expect derivative expansions to be well behaved [4] at least in many situations of interest, while derivative expansions automatically yield a sequence of systematic approximations (since there is expansion in only one 'parameter'). Additionally, since a derivative expansion in effect incorporates at each new level of approximation a new infinite set of operators?, these expansions provide the limits of reliability and accuracy that can anyway be reasonably hoped for from truncations to any finite set of operators.

The lowest order in the derivative expansion, ' $O\left(\partial^{0}\right)^{\prime}$, corresponds to the already well established Local Potential Approximation [9], in which the effective interactions are restricted to that of a general potential, and has proved reliable and reasonably accurate in a large variety of circumstances (see the references collected in [4, 10], and refs. [3, 8, [11]-[16]). $O\left(\partial^{2}\right)$ - second order in the derivative expansion, has been explored in [5], 17]-21]. The derivative expansion for the Legendre effective action with infrared cutoff (this being the one-particle irreducible part of the Wilson/Polchinski effective actions [2]) preserves more of

\footnotetext{
${ }^{1}$ in bosonic theories, corresponding to all powers of the fields
} 
the structure [2, [4] (e.g. preservation of quantization of anomalous scaling dimensions after derivative expansion, for certain cutoffs [19, 10, 14, as we review later). Very encouraging accuracy has been found in the results with this method for the non-perturbative massless quantum field theories of a single scalar field, corresponding to the two and three dimensional Ising model fixed points [19, 20], and the infinite sequence of two dimensional multicritical fixed points [20]. These latter results are particularly significant since these calculations lie well outside the practical capabilities of all other standard approximation methods (including lattice methods) 20]. Excellent results have also been found for the massive quantum field theory defined about three dimensional Ising model fixed point [5].

Here we extend these second order calculations to the non-perturbative massless $O(N)$ scalar field theories in three dimensions, corresponding to the WilsonFisher fixed points [回]. We remind the reader that the following values of $N$ are experimentally realised in critical phenomena: $N=0$ corresponding to polymers; $N=1$ corresponding to 'classical' critical liquid-vapour phase transitions, alloy order-disorder transitions, and uniaxial (Ising) ferromagnets; $N=2$ corresponding to the $\mathrm{He}^{2}$ superfluid phase transition, and to planar ferromagnets; $N=3$ corresponding to (Heisenberg) ferromagnets; and $N=4$ corresponding to the chiral phase transition for two quark flavours [22, 23].

Our results for these cases, compared to the worlds best present estimates, are fair for the lowest order, and already rather good ${ }^{2}$ at second order of approximation. As $N$ increases beyond four however, the results for $\eta$ particularly become poorer but we find good results for $\omega$, until as $N \rightarrow \infty$, we achieve the exact results for all exponents [11, 22]. We show also that at $N=-2,-4, \cdots$, the results from derivative expansion coincide with the known exact results [24, 25, 26].

At $N=\infty$, we give explicit analytic solutions for the fixed point effective potential, and the eigenperturbations in the potential and kinetic terms. We obtain analytically all the corresponding eigenvalues, even though the perturbations to the kinetic term actually lie outside the exactly soluble sector in large $N$ methods [16]. At the special values $N=-2,-4, \cdots$, we obtain analytically the known exact eigenvalues but also find intruiging hints for some novel fixed points.

Most importantly in our opinion, we continue to find (for all $N$ ), that the derivative expansion is qualitatively reliable - in the sense that no spurious fixed points or eigenvalues are found (in contrast to the generic situation for finite numbers of operators [3, 27]).

The organisation of the paper is as follows. In section 2 we review the method as set up in [19], and provide the slight extensions necessary for the $N$ compo-

\footnotetext{
${ }^{2}$ with the exception of $\omega$ when $N>1$
} 
nent case. In section 3 we develop the $O\left(\partial^{0}\right)$ approximation. We remind the reader why the only approximation that actually need be made is to truncate the derivative expansion of the full connected two-point function. We derive the boundary conditions required for the fixed point and eigenperturbations. In section 4 we develop the $O\left(\partial^{2}\right)$ approximation, again develop the relevant boundary conditions, identify the one redundant operator expected, and discuss the special behaviour at $N=1$. Section 5 deals with the case $N=\infty$. We here derive the exact explicit solutions already mentioned above, and identify in passing, the line of Gaussian fixed points that appear at $N=\infty$, and the end-point responsible for the Bardeen-Mosher-Bander phenomenon. We point out here the possibility to search for fixed points lieing outside the exactly soluble sector in large $N$ methods, and provide physical justification for our analytic expressions for the eigenvalues for the kinetic term perturbations, which also lie outside the exactly soluble sector. In section 6 , we show how to recover at $N=-2$ and $N=-4$, the known exact results for certain eigenvalues. At the same time we find at $N=-4$, that there might exist a non-trivial fixed point with two exactly soluble irrational eigenvalues. A similar pattern is expected for the other negative even $N$. Finally, in section 7 we present and discuss the numerical results. In deriving these, a number of numerical problems had to be understood and overcome, as described in appendix A. Appendix B provides some necessary beyond leading order asymptotic expressions.

\section{Review}

In this section we review, extending slightly where necessary, the derivative expansion method developed in [19. We take the following as our partition function,

$$
\exp W[J]=\int \mathcal{D} \varphi \exp \left\{-\frac{1}{2} \varphi \cdot C^{-1} \cdot \varphi-S_{\Lambda_{0}}[\varphi]+J \cdot \varphi\right\} \quad .
$$

We use a condensed notation, so that two-point functions are regarded as matrices in position or momentum space, as well as any internal index space; one-point functions as vectors; and contractions indicated by a dot. We will work in $D$ Euclidean dimensions with a real scalar field with $N$ components: $\varphi^{a}, a=1, \cdots, N$. $S_{\Lambda_{0}}$ is the full bare action for the theory, and is taken to have an internal $O(N)$ symmetry. $C \equiv C(q, \Lambda)$ is taken to be an additive cutoff for convenience 19, satisfying $C \rightarrow 0$ as $q \rightarrow 0$, and $q^{2} C(q, \Lambda) \rightarrow \infty$ as $q \rightarrow \infty$.

From (11) we have

$$
\frac{\partial}{\partial \Lambda} W[J]=-\frac{1}{2}\left\{\frac{\delta W}{\delta J} \cdot \frac{\partial C^{-1}}{\partial \Lambda} \cdot \frac{\delta W}{\delta J}+\operatorname{tr}\left(\frac{\partial C^{-1}}{\partial \Lambda} \cdot \frac{\delta^{2} W}{\delta J \delta J}\right)\right\}
$$


Re-writing this in terms of the Legendre effective action, $\Gamma_{\Lambda}$, defined by $\Gamma_{\Lambda}\left[\varphi^{c}\right]+\frac{1}{2} \varphi^{c} \cdot C^{-1} \cdot \varphi^{c}=-W[J]+J \cdot \varphi^{c}$, where the classical field $\varphi^{c}=\delta W / \delta J$, gives

$$
\frac{\partial}{\partial \Lambda} \Gamma_{\Lambda}\left[\varphi^{c}\right]=-\frac{1}{2} \operatorname{tr}\left[\frac{1}{C} \frac{\partial C}{\partial \Lambda} \cdot\left(1+C \cdot \frac{\delta^{2} \Gamma_{\Lambda}}{\delta \varphi^{c} \delta \varphi^{c}}\right)^{-1}\right] .
$$

The trace represents a sum over the internal spin indices and an integration over momentum/position space. As in ref. [2] it is convenient to write the trace as an integral over momentum space and factor out the $D$-dimensional solid angle. At the same time, we drop the superscript ${ }^{c}$ on the classical field:

$$
\frac{\partial \Gamma_{\Lambda}[\varphi]}{\partial \Lambda}=-\frac{\Omega}{2} \operatorname{tr} \int_{0}^{\infty} d q \frac{q^{D-1}}{C(q, \Lambda)} \frac{\partial C(q, \Lambda)}{\partial \Lambda}\left\langle\left[1+C \cdot \frac{\delta^{2} \Gamma_{\Lambda}}{\delta \varphi \delta \varphi}\right]^{-1}(\mathbf{q},-\mathbf{q})\right\rangle
$$

where $\Omega=2 /\left[\Gamma(D / 2)(4 \pi)^{D / 2}\right]$ is the solid angle of a $D$ - 1-dimensional sphere divided by $(2 \pi)^{D}$, the brackets $\langle\cdots\rangle$ represent an average over all directions of the momentum $\mathbf{q}$, and the trace now represents only the trace over the internal indices.

At a fixed point, the field $\varphi$ scales anomalously as $\varphi \sim \Lambda^{D_{\varphi}}$, where $D_{\varphi}=$ $\frac{1}{2}(D-2+\eta)$ and $\eta$ is the anomalous scaling dimension. Considering the defining expression for $\Gamma_{\Lambda}, \Gamma_{\Lambda}[\varphi]=-\frac{1}{2} \varphi \cdot C^{-1} \cdot \varphi-W_{\Lambda}[J]+J . \varphi$, dimensional analysis then shows that we require $C$ to behave as follows,

$$
C(q, \Lambda) \rightarrow \Lambda^{\eta-2} \tilde{C}\left(q^{2} / \Lambda^{2}\right)
$$

for some $\tilde{C}$, if we are to express $\Gamma_{\Lambda}$ as independent of $\Lambda$ at the fixed point. From now on we write $C(q, \Lambda)$ as $\Lambda^{\eta-2} \tilde{C}\left(q^{2} / \Lambda^{2}\right)$ and drop the tilde on the scaled $C$. As we are only interested in the behaviour near or at fixed points this is a sensible definition and we can take $\eta$ to be a constant. This is the reason for choosing an additive cutoff: a multiplicative cutoff $C_{I R}$ cannot scale homogeneously (to absorb $\Lambda^{\eta}$ ), and remain consistent with its normalisation in the ultraviolet (viz. $C_{I R} \rightarrow 1$ as $q \rightarrow \infty$ ) [19]. Now it is necessary (for $\Gamma_{\Lambda}$ to be independent of $\Lambda$ at fixed points) to re-write the equations in terms of dimensionless variables,

$$
\begin{aligned}
\mathbf{q} & \rightarrow \Lambda \mathbf{q} \\
\varphi(\Lambda \mathbf{q}) & \rightarrow \Lambda^{D-D_{\varphi}} \varphi(\mathbf{q}),
\end{aligned}
$$

and we define $t=\log (\mu / \Lambda)$ (where $\mu$ is an arbitrary finite energy scale). Finally we rescale the fields and the effective action to absorb the factor of $\Omega / 2$ in (国),

$$
\begin{aligned}
\Gamma_{t} & \rightarrow \frac{\Omega}{2 \zeta} \Gamma_{t} \\
\varphi^{a} & \rightarrow \sqrt{\frac{\Omega}{2 \zeta}} \varphi^{a}
\end{aligned}
$$


where $\zeta$ is a numerical normalization factor to be chosen for later convenience. Upon doing this we get,

$$
\begin{aligned}
& \left(\frac{\partial}{\partial t}+D_{\varphi} \Delta_{\varphi}+\Delta_{\partial}-D\right) \Gamma_{t}[\varphi]= \\
& \quad-\zeta \operatorname{tr} \int_{0}^{\infty} d q q^{D-1}\left(\frac{q}{C\left(q^{2}\right)} \frac{\partial C\left(q^{2}\right)}{\partial q}+2-\eta\right)\left\langle\left[1+C \cdot \frac{\delta^{2} \Gamma_{t}}{\delta \varphi \delta \varphi}\right]^{-1}(\mathbf{q},-\mathbf{q})\right\rangle
\end{aligned}
$$

In the above $\Delta_{\varphi}=\varphi \cdot \frac{\delta}{\delta \varphi}$ is the field counting operator: it counts the number of occurrences of the field $\varphi$ in a given vertex, and arises due to the scaling of the field in (6). $\Delta_{\partial}$ is the momentum counting operator plus the dimension of space $D$ and arises through the rescaling of the momenta in equation (6). It can be represented as

$$
\Delta_{\partial}=D+\int \frac{d^{D} p}{(2 \pi)^{D}} \varphi(\mathbf{p}) p^{\mu} \frac{\partial}{\partial p^{\mu}} \frac{\delta}{\delta \varphi(\mathbf{p})}
$$

Operating on a given vertex it counts the total number of derivatives acting on the fields $\varphi$. Equation (7) will be the starting point for all the work from now onwards. Notice that for the first time $\eta$ explicitly appears in the equation.

We write $\Gamma_{t}[\varphi]$ as the space-time integral of an effective Lagrangian expanded in powers of derivatives,

$$
\Gamma_{t}[\varphi]=\int d^{D} x\left\{V\left(\varphi^{2}, t\right)+\frac{1}{2} K\left(\varphi^{2}, t\right)\left(\partial_{\mu} \varphi^{a}\right)^{2}+\frac{1}{2} Z\left(\varphi^{2}, t\right)\left(\varphi^{a} \partial_{\mu} \varphi^{a}\right)^{2}+\cdots\right\}
$$

Each linearly independent (under integration by parts) combination of differentiated fields will carry its own general (t-dependent) coefficient. The global $O(N)$ symmetry forces us to choose the coefficient functions to be functions of $\varphi^{2}$. We will require that the fixed point solutions for $V, K, Z$ etc. will be nonsingular for all $\varphi^{2}$, that perturbations about these solutions grow no faster than a power 14,5 , 田, and that $K(0) \neq 0$. It will be seen that the imposition of power law growth results in a quantized spectrum.

It was realized some time ago that a general cutoff function $C\left(q^{2}\right)$, for example based upon an exponential function, leaves $\eta$ undetermined and dependent upon an unphysical parameter 18, 4]. However, dimensional analysis indicates that if $C\left(q^{2}\right)$ is chosen to be homogenous in $q^{2}$ then equation (17) is invariant under a global rescaling symmetry [19]. This rescaling symmetry overdetermines the equations, so that solutions only exist for discrete values of $\eta$. We will follow this path and take $C\left(q^{2}\right)=q^{2 k}$, for $k$ a positive integer. From general arguments it can be shown that the convergence is slower the higher the value of $k$ [2, 19]. 
On the other hand, if we wish the integrals in equation (7) to be ultra-violet convergent we must take $k>D / 2-1$ [19]. Therefore we take $k$ to be the smallest integer greater than $D / 2-1$, so for $D=3$ we take $k=1$. The rescaling symmetry is made explicit by choosing the following (non-physical) scaling dimensions, as follows from (9) and the definition of $\Gamma_{t}$.

$$
\begin{array}{lcl}
{\left[q^{\mu}\right]=1} & {\left[\partial^{\mu}\right]=1} & {\left[\varphi^{a}\right]=k+D / 2} \\
{[V]=D} & {[K]=-2(k+1)} & {[Z]=-2(2 k+1)-D}
\end{array} .
$$

The expansion is performed by substituting equation (9) into equation (77) and expanding the right hand side up to a maximum number of derivatives. The angular average in (7) can be easily computed by translating them into invariant tensors, e.g. $\left\langle q^{\mu} q^{\nu}\right\rangle=q^{2} \delta^{\mu \nu} / D$ etc. From now on we will specialize to $D=3$.

\section{Lowest Order of Approximation}

To lowest order in the expansion we drop all the derivatives from the right hand side of (7). At the same time, a reasonable ansatz for $\Gamma_{t}$ would be to keep only a general potential $V\left(\varphi^{2}, t\right)$ in (9), setting $K=1$, and $Z$ etc. to zero. It is worthwhile to remark however, that there is no need for a further ansatz beyond that of dropping all derivative terms from the right hand side of (7), i.e. from the full connected two-point function (and similarly at higher orders of the derivative expansion): the form of $\Gamma_{t}$ is then determined by consistency arguments [19]. Substituting (9) into (17), we see that the coefficient functions $K, Z$, etc. are determined by linear equations given by the vanishing of the left hand side of (7). Consider the equation for $K$,

$$
\frac{\partial}{\partial t} K\left(\varphi^{2}, t\right)+(1+\eta) \varphi^{2} K^{\prime}\left(\varphi^{2}, t\right)+\eta K\left(\varphi^{2}, t\right)=0
$$

where ${ }^{\prime}=\frac{\partial}{\partial\left(\varphi^{2}\right)}$. We see that at fixed points, where $K$ is independent of $t$,

$$
K\left(\varphi^{2}\right) \propto\left(\varphi^{2}\right)^{-\eta /(1+\eta)}
$$

If $K\left(\varphi^{2}\right)$ is to be non-singular (at $\varphi=0$ ) and $K(0) \neq 0$, we must have $\eta=0$. Therefore $K\left(\varphi^{2}\right)$ is a constant. Using the scaling symmetry we can set this constant to be $K=1$. If we now consider the equation for $Z$ we get

$$
\frac{\partial}{\partial t} Z\left(\varphi^{2}, t\right)+\varphi^{2} Z^{\prime}\left(\varphi^{2}, t\right)+Z\left(\varphi^{2}, t\right)=0
$$

Hence we see that at fixed points, $Z$ will satisfy

$$
Z \propto\left(\varphi^{2}\right)^{-1}
$$


To obtain non-singular behaviour, we must set the constant of proportionality to zero and thus $Z\left(\varphi^{2}\right) \equiv 0$. Considering the form of a general term in the expansion we see that a similar conclusion must hold for all other terms. A general term, $H$, will satisfy

$$
\frac{\partial}{\partial t} H\left(\varphi^{2}, t\right)+\varphi^{2} H^{\prime}\left(\varphi^{2}, t\right)+n_{\varphi} H\left(\varphi^{2}, t\right)+n_{\partial} H\left(\varphi^{2}, t\right)-3 H\left(\varphi^{2}, t\right)=0 \quad,
$$

where $n_{\varphi}$ is the number of $\varphi$ 's occurring in the term multiplying $H$, divided by two and $n_{\partial}$ is the number of derivatives occurring in the term multiplying $H$. Hence we see that at a fixed point, we have

$$
H \propto\left(\varphi^{2}\right)^{-\left(n_{\varphi}+n_{\partial}-3\right)}
$$

Thus for terms of higher order in the expansion, where $n_{\varphi} \geq 2$ and $n_{\partial}>2, H$ will be singular unless the constant of proportionality is set to zero. Therefore, at leading order, the derivative expansion must reproduce the local potential expansion at fixed points, and thus we take

$$
\Gamma_{t}[\varphi]=\int d^{D} x V\left(\varphi^{2}, t\right)+\frac{1}{2}\left(\partial_{\mu} \varphi^{a}\right)^{2}
$$

Since we discard all derivatives from $\left[1+C \cdot \frac{\delta^{2} \Gamma_{t}[\varphi]}{\delta \varphi \delta \varphi}\right]^{-1}(\mathbf{q},-\mathbf{q})$, we have that $\varphi$ is effectively a constant and the operator diagonal in momentum space (as explained further in the next section), thus it is the matrix inverse of

$$
\delta^{a b}+C\left(q^{2}\right) \frac{\delta^{2} \Gamma_{t}[\varphi]}{\delta \varphi^{a}(-\mathbf{q}) \delta \varphi^{b}(\mathbf{q})}=\delta^{a b}+q^{2}\left(2 \delta^{a b} V^{\prime}+4 \varphi^{a} \varphi^{b} V^{\prime \prime}+q^{2} \delta^{a b}\right)
$$

The inverse and trace in $(\overline{7})$, are now easily accomplished by noting that a matrix $A \delta^{a b}+B \varphi^{a} \varphi^{b}$ has $N-1$ eigenvalues $A$ and one eigenvalue $A+\varphi^{2} B$ :

$$
\begin{aligned}
& \frac{\partial V\left(\varphi^{2}, t\right)}{\partial t}+\varphi^{2} V^{\prime}\left(\varphi^{2}, t\right)-3 V\left(\varphi^{2}, t\right)= \\
& \quad-4 \zeta \int_{0}^{\infty} d q q^{2} \frac{N-1}{1+2 V^{\prime}\left(\varphi^{2}, t\right) q^{2}+q^{4}}+\frac{1}{1+\left(2 V^{\prime}\left(\varphi^{2}, t\right)+4 \varphi^{2} V^{\prime \prime}\left(\varphi^{2}, t\right)\right) q^{2}+q^{4}}
\end{aligned}
$$

Performing the $q$-integrals, and setting $\zeta$ to $1 / 2 \pi$ yields the equation at leading order,

$$
\begin{aligned}
\frac{\partial V(\varphi, t)}{\partial t}+\varphi^{2} V^{\prime}\left(\varphi^{2}, t\right)-3 V\left(\varphi^{2}, t\right)= & -\frac{1}{\sqrt{2+2 V^{\prime}\left(\varphi^{2}, t\right)+4 \varphi^{2} V^{\prime \prime}\left(\varphi^{2}, t\right)}} \\
& -\frac{N-1}{\sqrt{2+2 V^{\prime}\left(\varphi^{2}, t\right)}} .
\end{aligned}
$$


Let us now discuss fixed point solutions of (20), that is solutions with $\partial V / \partial t=$ 0 . At first sight it may seem that (20) has infinitely many fixed point solutions. In fact this is not the case, as only finitely many solutions do not end in a singularity 14, 14. Of course this is sensible on physical grounds, as the fixed points correspond to massless continuum limits (i.e. continuous phase transitions) with the prescribed field content. Considering the form of (20) we see that either $V\left(\varphi^{2}\right)$ is only defined for $\varphi^{2}<\varphi_{c}^{2}$ and ends at a singularity, at $\varphi^{2}=\varphi_{c}^{2}$ as follows,

$$
\frac{1}{\varphi_{c}^{2}}\left(\frac{9}{16}\right)^{2 / 3}\left(\varphi_{c}^{2}-\varphi^{2}\right)^{4 / 3}
$$

(where we have suppressed non-singular and lower order singular parts), which we disregard as unphysical, or it is defined for all $\varphi^{2}>0$, and in particular for $\varphi^{2} \rightarrow \infty$. In this regime, we find that either $V\left(\varphi^{2}\right)$ is just a constant (the Gaussian fixed point), or $V\left(\varphi^{2}\right)$ must satisfy the following,

$$
A_{v}\left(\varphi^{2}\right)^{3}+\left(N+\frac{1}{\sqrt{5}}-1\right) \frac{1}{4 \sqrt{6 A_{v}} \varphi^{2}}+O\left(\left(\varphi^{2}\right)^{-2}\right)
$$

for some constant $A_{v}$. If we consider linear perturbations about this solution we see that for large $\varphi^{2}$ the perturbation must behave as a linear combination of $\left(\varphi^{2}\right)^{3}$ and $\exp \left(\frac{1}{8}\left[30 A_{v}\right]^{3 / 2}\left(\varphi^{2}\right)^{4}\right)$. The first perturbation merely alters the value of $A_{v}$, whilst we disallow the second as it is incompatible with (22). We see that, apart from the Gaussian fixed point, the solution space of fixed point solutions defined for all $\varphi^{2}$, forms an isolated one parameter set, parametrized by the value of $A_{v}$. Since we require $V\left(\varphi^{2}\right)$ also to be non-singular at the origin, setting $\varphi^{2}=0$ in equation (20) we obtain,

$$
-3 V(0)=-\frac{N}{\sqrt{2+2 V^{\prime}(0)}}
$$

We now have a second order differential equation with two boundary conditions: (22,23). Thus we expect at most a discrete set of acceptable solutions. In fact we only find two:- the Gaussian fixed point, $V\left(\varphi^{2}\right)=\frac{N}{3 \sqrt{2}}$, and an approximation to the Wilson-Fisher fixed point [1]. To find this non-trivial solution we need to rely on numerical methods, as outlined in the appendix A. The results of these calculations are shown in figure 1, for various values of $N$.

To calculate the critical exponents, we linearize about this fixed point potential $V=V^{*}\left(\varphi^{2}\right)$. Writing $V\left(\varphi^{2}, t\right)=V^{*}\left(\varphi^{2}\right)+\delta V\left(\varphi^{2}, t\right)$, where $\delta V\left(\varphi^{2}, t\right)$ is given by $\varepsilon \mathrm{e}^{\lambda t} v\left(\varphi^{2}\right)$, with $\varepsilon \ll 1$, and expanding to first order in $\varepsilon$, we have

$$
(\lambda-3) v\left(\varphi^{2}\right)+\varphi^{2} v^{\prime}\left(\varphi^{2}\right)=\frac{(N-1) v^{\prime}\left(\varphi^{2}\right)}{\left(2+2 V^{\prime}\left(\varphi^{2}\right)\right)^{3 / 2}}+\frac{v^{\prime}\left(\varphi^{2}\right)+2 \varphi^{2} v^{\prime \prime}\left(\varphi^{2}\right)}{\left(2+2 V^{\prime}\left(\varphi^{2}\right)+4 \varphi^{2} V^{\prime \prime}\left(\varphi^{2}\right)\right)^{3 / 2}}
$$




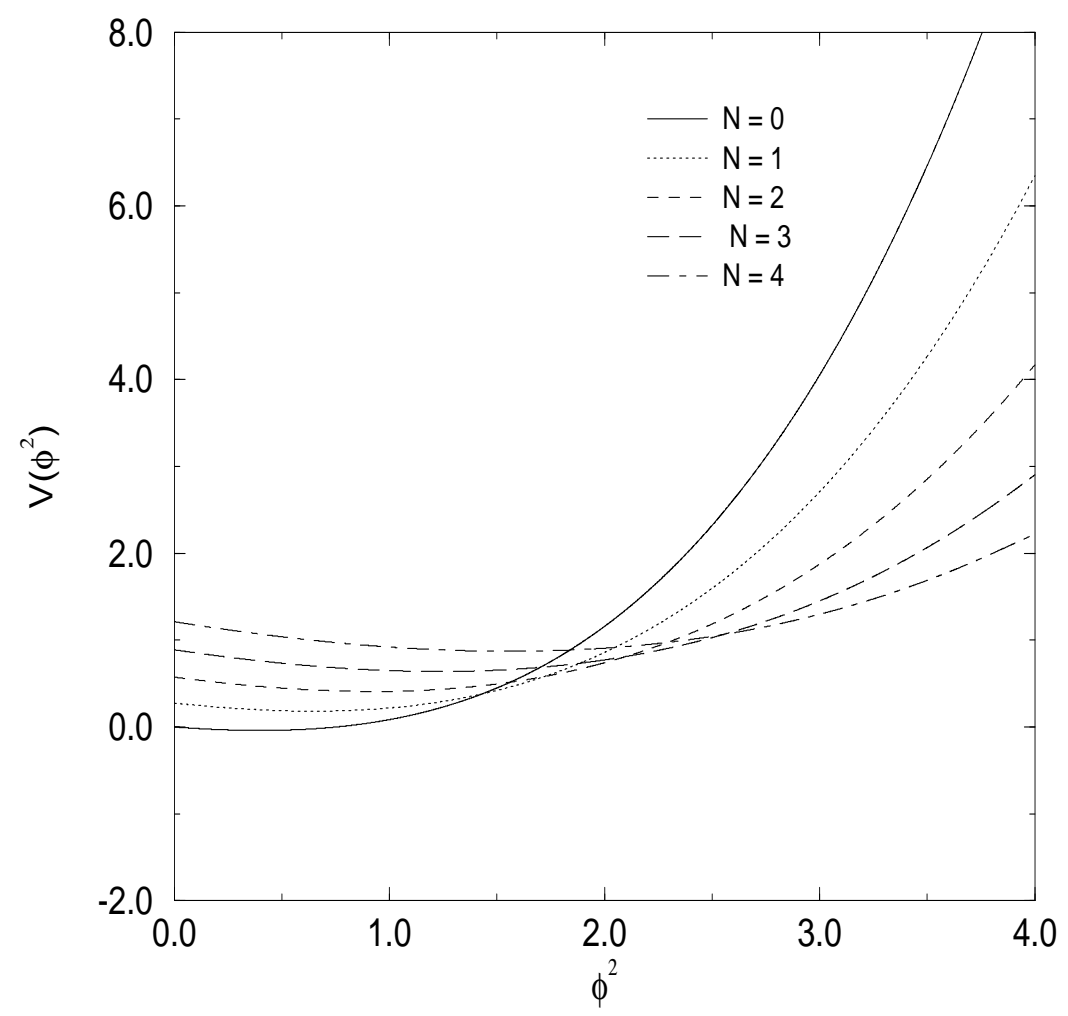

Figure 1: The potential at leading order in the derivative expansion.

Requiring that $v\left(\varphi^{2}\right)$ is not singular at the origin $\varphi^{2}=0$, we obtain

$$
(\lambda-3) v(0)=\frac{N}{\left(2+2 V^{\prime}(0)\right)^{3 / 2}} v^{\prime}(0) \quad .
$$

Thus for $N \neq 0$ we may choose, by linearity of the perturbation, the normalisation condition $v(0)=1$. For $N=0$, (25) implies that either $\lambda=3$ or $v(0)=0$. We discard the case of $\lambda=3$ as this corresponds to the uninteresting case of the vacuum energy operator $v\left(\varphi^{2}\right) \equiv 1$. Therefore for $N=0$ one has $v(0)=0$, and by linearity we can take $v^{\prime}(0)=1$. Thus for any $N \geq 0$, we obtain two boundary conditions at the origin. For large $\varphi$, we see that the perturbation will be a linear combination of

$$
\left(\varphi^{2}\right)^{3-\lambda}
$$

and $\exp \left(\frac{1}{8}\left[30 A_{v}\right]^{3 / 2}\left(\varphi^{2}\right)^{4}\right)$. Once more we will enforce a coefficient of zero on the latter perturbation, as we require the perturbations to grow no faster than a power in $\varphi^{2}$ [14, 毘, The imposition of this condition will ensure that the solutions form an isolated one parameter set. Upon also imposing the two boundary conditions at the origin, we expect at most a discrete number of solutions to the eigenvalue 
problem for $\lambda$, which is indeed the case. We find only one positive eigenvalue, which yields $\nu$ through $\nu=1 / \lambda$. The least negative eigenvalue yields the first correction to scaling through $\omega=-\lambda$. The results are summarized in the table presented in section 7 .

\section{Second Order of Approximation}

The equations at the second order in the expansion are calculated by substituting expression (9) into equation (7) and dropping all terms with more than two derivatives from the right hand side. Following a similar argument to that given in the previous section, we see that terms with more than two derivatives are forced to vanish, hence at second order of the derivative expansion, only the terms explicitly written in (9) survive. A little bit of thought shows that a similar conclusion holds at all higher orders of the approximation: if we substitute an expression into the equation that is of higher order than the order we are working at, then we are forced to set the higher order terms to zero [19].

The computation of the inverse in equation (ब), is not as straightforward as in the leading order case. We regard $\left[1+C . \delta^{2} \Gamma_{t} / \delta \varphi \delta \varphi\right]^{-1}$ as a differential operator:

$$
\begin{aligned}
{\left[1+C \cdot \frac{\delta^{2} \Gamma_{t}}{\delta \varphi \delta \varphi}\right]^{-1}(\mathbf{q},-\mathbf{q}) } & =\int d^{D} x d^{D} y \mathrm{e}^{-i \mathbf{q} \cdot \mathbf{x}}\left[\delta^{a b}+C \cdot \frac{\delta^{2} \Gamma_{t}}{\delta \varphi^{a} \delta \varphi^{b}}\right]^{-1}(\mathbf{x}, \mathbf{y}) \mathrm{e}^{i \mathbf{q} \cdot \mathbf{y}} \\
& \equiv \int d^{D} x Q \\
\text { where } \quad & =\mathrm{e}^{-i \mathbf{q} \cdot \mathbf{x}}\left[1+C \cdot \frac{\delta^{2} \Gamma_{t}}{\delta \varphi \delta \varphi}\right]^{-1} \mathrm{e}^{i \mathbf{q} \cdot \mathbf{x}} .
\end{aligned}
$$

$Q^{a b}$ is a function of $\mathbf{q}$ and $\varphi(\mathbf{x})$ and its derivatives evaluated at $\mathbf{x}$, and $C$ and $\frac{\delta^{2} \Gamma_{t}}{\delta \varphi^{a} \varphi^{b}}$ are written as differential operators: $C(-\square)$,

$$
\begin{aligned}
& \frac{\delta^{2} \Gamma}{\delta \varphi^{a}} \delta \varphi^{b}=4 \varphi^{a} \varphi^{b} V^{\prime \prime}+2 \delta^{a b} V^{\prime}-\delta^{a b} K \square-2\left(\square \varphi^{a}\right) \varphi^{b} K^{\prime}-2 \delta^{a b}\left(\varphi^{c} \partial_{\mu} \varphi^{c}\right) K^{\prime} \partial_{\mu} \\
& \quad-2\left(\partial_{\mu} \varphi^{a}\right)\left(\partial_{\mu} \varphi^{b}\right) K^{\prime}-2\left(\partial_{\mu} \varphi^{a}\right) \varphi^{b} K^{\prime} \partial_{\mu}-4\left(\partial_{\mu} \varphi^{a}\right) \varphi^{b}\left(\varphi^{c} \partial_{\mu} \varphi^{c}\right) K^{\prime \prime} \\
& \quad+2 \varphi^{a}\left(\partial_{\mu} \varphi^{b}\right) K^{\prime} \partial_{\mu}+\delta^{a b}\left(\partial_{\mu} \varphi^{c}\right)^{2} K^{\prime}+2 \varphi^{a} \varphi^{b}\left(\partial_{\mu} \varphi^{c}\right)^{2} K^{\prime \prime}-\delta^{a b}\left(\partial_{\mu} \varphi^{c}\right)^{2} Z \\
& \quad-2 \varphi^{a}\left(\partial_{\mu} \varphi^{b}\right) Z \partial_{\mu}-2 \varphi^{a} \varphi^{b}\left(\partial_{\mu} \varphi^{c}\right)^{2} Z^{\prime}-\delta^{a b}\left(\varphi^{c} \square \varphi^{c}\right) Z-\varphi^{a}\left(\square \varphi^{b}\right) Z \\
& \quad-\varphi^{a} \varphi^{b} Z \square-2 \varphi^{a} \varphi^{b}\left(\varphi^{c} \square \varphi^{c}\right) Z^{\prime}-\delta^{a b}\left(\varphi^{c} \partial_{\mu} \varphi^{c}\right)^{2} Z^{\prime} \\
& \quad-2 \varphi^{a}\left(\partial_{\mu} \varphi^{b}\right)\left(\varphi^{c} \partial_{\mu} \varphi^{c}\right) Z^{\prime}-2 \varphi^{a} \varphi^{b}\left(\varphi^{c} \partial_{\mu} \varphi^{c}\right) Z^{\prime} \partial_{\mu}-2 \varphi^{a} \varphi^{b}\left(\varphi^{c} \partial_{\mu} \varphi^{c}\right)^{2} Z^{\prime \prime}
\end{aligned}
$$

Define $\nu^{a b}$ as the expression obtained by dropping all terms containing differentials 
of $\varphi$ from $C\left(q^{2}\right) \mathrm{e}^{-i \mathbf{q} \cdot \mathbf{x}} \frac{\delta^{2} \Gamma}{\delta \varphi^{a} \delta \varphi^{b}} \mathrm{e}^{i \mathbf{q} \cdot \mathbf{x}}:$

$$
\nu^{a b}=q^{2}\left(4 \varphi^{a} \varphi^{b} V^{\prime \prime}+2 \delta^{a b} V^{\prime}+\delta^{a b} K q^{2}+\varphi^{a} \varphi^{b} Z q^{2}\right) .
$$

(Recall $C\left(q^{2}\right)=q^{2}$ in the present case.) Noting that from (28),

$$
Q^{a b}=\delta^{a b}-\mathrm{e}^{-i \mathbf{q} \cdot \mathbf{x}} C(-\square) \frac{\delta^{2} \Gamma}{\delta \varphi^{a} \delta \varphi^{c}}\left(\mathrm{e}^{i \mathbf{q} \cdot \mathbf{x}} Q^{c b}\right)
$$

we see that $Q^{a b}$ satisfies the following expression [19,

$$
Q^{a b}=(1+\nu)_{a b}^{-1}+(1+\nu)_{a c}^{-1}\left\{\nu^{c d} Q^{d b}-\mathrm{e}^{-i \mathbf{q} \cdot \mathbf{x}} C(-\square) \frac{\delta^{2} \Gamma}{\delta \varphi^{c} \delta \varphi^{d}}\left(\mathrm{e}^{i \mathbf{q} \cdot \mathbf{x}} Q^{d b}\right)\right\}
$$

(as may be verified by multiplying by $\delta^{e a}+\nu^{e a}$ ). By construction, the term in curly brackets in (32) is at least of first order in the derivative expansion. The derivative expansion to any order may thus be derived by iterating expression (32), starting with the zeroth order $Q^{a b}=(1+\nu)_{a b}^{-1}$.

We iterated just to two derivatives [using the fact that $1+\nu$ is of the form $\delta^{a b}+\nu^{a b}=A \delta^{a b}+B \varphi^{a} \varphi^{b}$, so $(1+\nu)_{a b}^{-1}=\frac{\delta^{a b}}{A}-\frac{B}{A\left(A+B \varphi^{2}\right)} \varphi^{a} \varphi^{b}$, and the identity $\left.\partial_{\mu}(1+\nu)^{-1}=-(1+\nu)^{-1}\left(\partial_{\mu} \nu\right)(1+\nu)^{-1}\right]$. Even so, this is a long calculation, which we performed using the symbolic manipulation package Form [28]. Finally, in preparation for the $N=\infty$ limit, we scale the equations as follows [22, 16],

$$
\varphi=\sqrt{N} \tilde{\varphi}, \quad V=N \tilde{V}, \quad K=\tilde{K}, \quad Z=\tilde{Z} / N
$$

From the zeroth order part of $Q^{a a}$, we obtain

$$
\begin{aligned}
& \frac{\partial}{\partial t} V+(1+\eta) \varphi^{2} V^{\prime}-3 V=-(1-\eta / 4) \times \\
& \quad\left[\frac{1}{N \sqrt{K+\varphi^{2} Z} \sqrt{2 V^{\prime}+4 \varphi^{2} V^{\prime \prime}+2 \sqrt{K+\varphi^{2} Z}}}+\frac{1-1 / N}{\sqrt{K} \sqrt{2 V^{\prime}+2 \sqrt{K}}}\right]
\end{aligned}
$$

(where here and from now on we drop the tildes on $\varphi, V, K$ and $Z$ ). Similarly, from the $\left(\partial_{\mu} \varphi^{c}\right)^{2}$ part of $Q^{a a}$ we obtain,

$$
\begin{aligned}
& \frac{\partial}{\partial t} K+(1+\eta) \varphi^{2} K^{\prime}+\eta K= \\
& \quad \frac{(4-\eta)}{\pi}\left[I_{4,2,1}\left(\frac{4}{3} \frac{K^{\prime 2} \varphi}{N}+\frac{4}{3} \frac{K^{\prime} Z \varphi}{N}\right)+I_{4,1,2}\left(\frac{4}{3} \frac{K^{\prime 2} \varphi}{N}-4 \frac{K^{\prime} Z \varphi}{N}+\frac{8}{3} \frac{Z^{2} \varphi}{N}\right)\right. \\
& \quad+\frac{16}{3} \frac{I_{3,2,1} V^{\prime \prime} K^{\prime} \varphi}{N}+I_{3,1,2}\left(-\frac{16}{3} \frac{V^{\prime \prime} K^{\prime} \varphi}{N}+\frac{16}{3} \frac{V^{\prime \prime} Z \varphi}{N}\right) \\
& \left.+I_{2,0,2}\left(\frac{K^{\prime}}{N}-\frac{Z}{N}-K^{\prime}\right)+I_{2,2,0}\left(-\frac{K^{\prime}}{N}-2 \frac{K^{\prime \prime} \varphi}{N}\right)\right]
\end{aligned}
$$


where for compactness we have defined the integrals

$$
I_{j, k, m}=\int_{0}^{\infty} \frac{q^{2 j} d q}{\left(1+\left(2 V^{\prime}+4 \varphi^{2} V^{\prime \prime}\right) q^{2}+\left(K+\varphi^{2} Z\right) q^{4}\right)^{k}\left(1+2 V^{\prime} q^{2}+K q^{4}\right)^{m}}
$$

These integrals can be evaluated in closed form, but (35) would then be too long to display. Furthermore, as discussed in appendix A, it proves numerically not sensible to do so. Even in this compact form however, the flow equation for $Z$ (which follows from the $\left(\varphi^{c} \partial_{\mu} \varphi^{c}\right)^{2}$ part of $Q^{a a}$ ) is too long to display [29],

$$
\frac{\partial}{\partial t} Z+(1+\eta) \varphi^{2} Z^{\prime}+(1+2 \eta) Z=\cdots
$$

As in the previous section, we obtain the boundary conditions for fixed point solutions, from the requirement that these solutions be non-singular. Thus for large $\varphi^{2}$, either the solution is the trivial Gaussian fixed point $(\eta=0, V=$ $1 / 3 \sqrt{2}, K=1, Z=0$ ), or the solutions behave as follows,

$$
\begin{aligned}
V\left(\varphi^{2}\right) & \sim A_{v}\left(\varphi^{2}\right)^{\frac{3}{1+\eta}}+\cdots \\
K\left(\varphi^{2}\right) & \sim A_{k}\left(\varphi^{2}\right)^{\frac{-\eta}{1+\eta}}+\cdots \\
Z\left(\varphi^{2}\right) & \sim A_{z}\left(\varphi^{2}\right)^{-\frac{1+2 \eta}{1+\eta}}+\cdots .
\end{aligned}
$$

We can also study the perturbations about these solutions, and forcing that the perturbations grow no faster than a power, we see that solutions satisfying (38) to (40) form an isolated four-parameter set, that is including $\eta$. From the requirement that the solutions are non-singular at the origin, substituting $\varphi^{2}=0$ in (34) - (37), we now obtain three conditions. Using the scaling symmetry (11), we can impose one further condition. We take $K(0)=1$ (with other possible solutions being reached by using the reparametrization invariance). Thus with four conditions imposed on a four-parameter set, we expect only a discrete number of solutions, in particular for $\eta$. In this way, (34) - (37) at fixed points, form non-linear eigenvalue equations for $\eta$.

Again we only find two solutions:- the Gaussian point mentioned above and an approximation to the Wilson-Fisher fixed point. The results for $\eta$ are summarized in the table presented in section 7 , and the results for the fixed point solutions shown in figures 2 to 4 .

To calculate the other critical exponents (at second order of approximation) we write, 


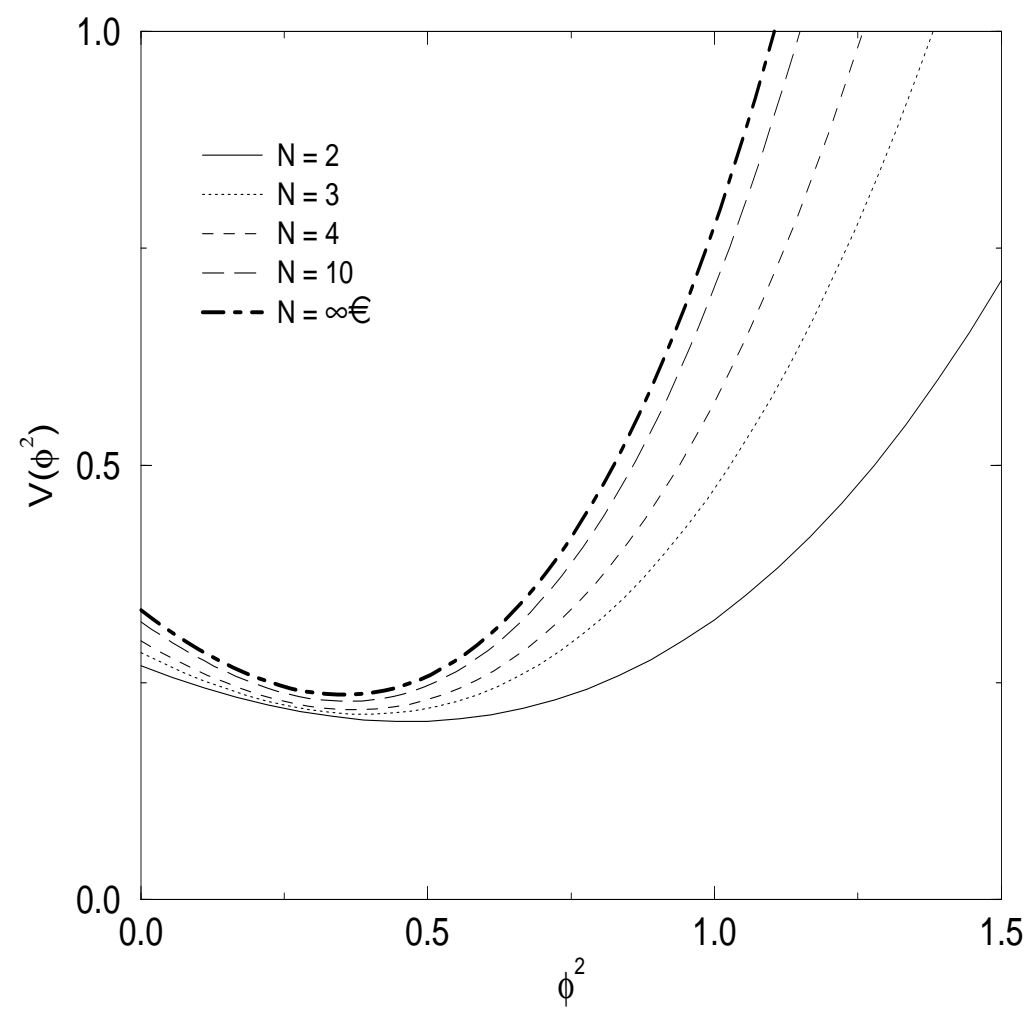

Figure 2: The potential at second order in the derivative expansion.

$$
\begin{aligned}
V\left(\varphi^{2}, t\right) & =V^{*}\left(\varphi^{2}\right)+\delta V\left(\varphi^{2}, t\right) \\
& =V^{*}\left(\varphi^{2}\right)+\varepsilon \mathrm{e}^{\lambda t} v\left(\varphi^{2}\right) \\
K\left(\varphi^{2}, t\right) & =K^{*}\left(\varphi^{2}\right)+\delta K\left(\varphi^{2}, t\right) \\
& =K^{*}\left(\varphi^{2}\right)+\varepsilon \mathrm{e}^{\lambda t} k\left(\varphi^{2}\right) \\
Z\left(\varphi^{2}, t\right) & =Z^{*}\left(\varphi^{2}\right)+\delta Z\left(\varphi^{2}, t\right) \\
& =Z^{*}\left(\varphi^{2}\right)+\varepsilon \mathrm{e}^{\lambda t} z\left(\varphi^{2}\right)
\end{aligned}
$$

where $V^{*}\left(\varphi^{2}\right), K^{*}\left(\varphi^{2}\right), Z^{*}\left(\varphi^{2}\right)$ (and $\eta^{*}$ ) are the fixed point solutions calculated above, and expand the equations to first order in $\varepsilon$. If we insist that $v, k$ and $z$ grow no faster than a power at large $\varphi^{2}$ [14, 5, , t], then asymptotic analysis shows that $v, k$ and $z$ grow according to their scaling dimension,

$$
\begin{aligned}
v\left(\varphi^{2}\right) & \sim a_{v}\left(\varphi^{2}\right)^{\frac{3}{1+\eta^{*}}-\lambda}+\cdots \\
k\left(\varphi^{2}\right) & \sim a_{k}\left(\varphi^{2}\right)^{\frac{-\eta^{*}}{1+\eta^{*}}-\lambda}+\cdots \\
z\left(\varphi^{2}\right) & \sim a_{z}\left(\varphi^{2}\right)^{-\frac{1+2 \eta^{*}}{1+\eta^{*}}-\lambda}+\cdots,
\end{aligned}
$$




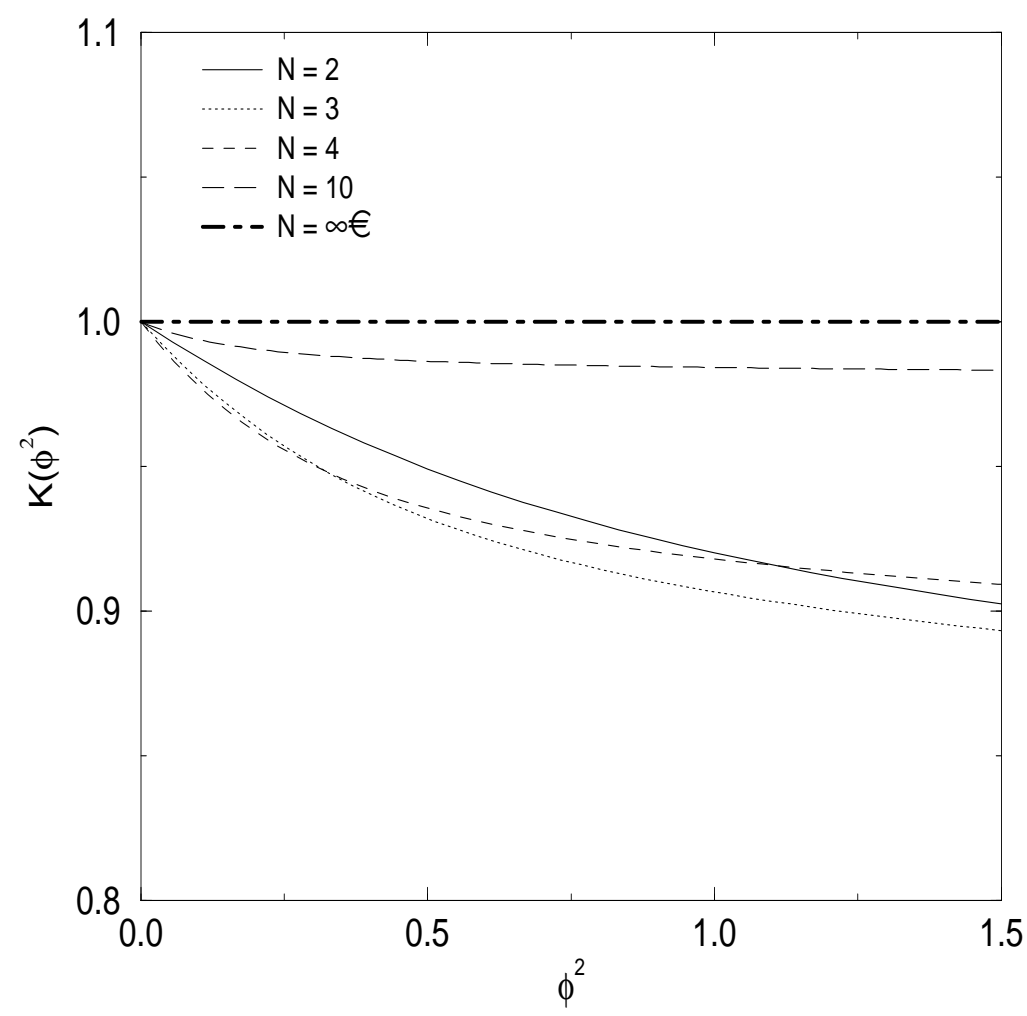

Figure 3: The $K$ component at second order in the derivative expansion.

and form an isolated four-parameter set (including $\lambda$ ). As before, another three boundary conditions come from substituting $\varphi^{2}=0$, assuming non-singular limits. Using linearity to set $v(0)=1$, we then expect at most a discrete set of solutions, which is what is found. Again we find just one positive eigenvalue, which yields $\nu$, and determine the least negative. These are shown in the table, presented in section 7 .

It is important to recognize that there are other solutions of the equations which do not correspond to critical indices [30]. These solutions are known as redundant perturbations and the eigenvalue corresponding to the solution depends on the exact form of the renormalization group chosen. The redundant perturbation reflects invariances of the equations [30, 19]. In fact we expect and find only one redundant perturbation corresponding to the reparametrization invariance (11). This has eigenvalue $\lambda=0$, and (not normalised)

$$
v=-5 \varphi^{2} V^{\prime}+3 V, \quad k=-5 \varphi^{2} K^{\prime}-4 K, \quad \text { and } \quad z=-5 \varphi^{2} Z^{\prime}-9 Z .
$$

An obvious question is why no redundant perturbations are found at the leading order. The answer is simple: the choice of $K \equiv 1$ (viz. for all $t$ ) breaks the 


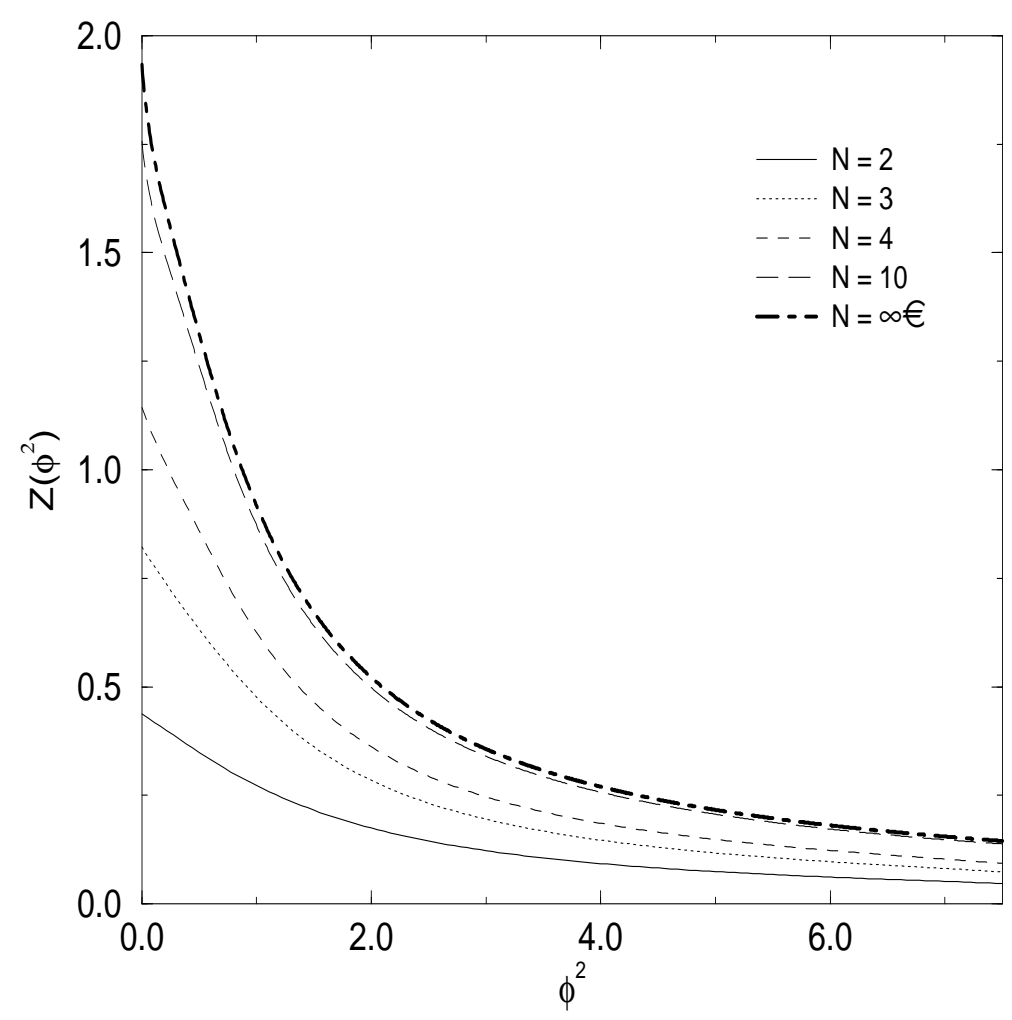

Figure 4: The $Z$ component at second order in the derivative expansion.

reparametrization invariance, thus we should not expect to see any redundant perturbation. Similarly we should not expect to see any perturbation corresponding to $\varphi$-translation invariance [30, 20] as the choice of $V, K$ and $Z$ being functions of $\varphi^{2}$ breaks this invariance.

The case of $N=1$ is special. Of course in this case there is only a discrete $Z_{2}$ internal symmetry, but also the derivative expansion (9) now becomes,

$$
\Gamma_{t}[\varphi]=\int d^{D} x\left\{V\left(\varphi^{2}, t\right)+\frac{1}{2}\left(K\left(\varphi^{2}, t\right)+\varphi^{2} Z\left(\varphi^{2}, t\right)\right)\left(\partial_{\mu} \varphi\right)^{2}\right\}
$$

We see that $Z$ and $K$ no longer have a separate significance, and we should consider only the function $\kappa=K+\varphi^{2} Z$. In fact at $N=1$, the $Z$ component of the fixed point solution to (34)-(37) is singular, diverging as $\sim 1 / \varphi^{2}$ as $\varphi^{2} \rightarrow 0$ (cf. appendix A). Taking (34), and forming $(35)+\varphi^{2}(37)$, we find that at $N=1$ all appearances of $K$ and $Z$ can be absorbed into $\kappa$, after which they coincide with the already analysed equations in ref. [19], as expected. 


\section{$5 \quad$ Exact Results at $N=\infty$}

It is known that in the large $N$ limit, the local potential approximation can effectively become exact [11, 8, 15, 16], in the sense that (17) then coincides with an exactly soluble subsector of the full flow equations. In this section we will show that the derivative expansion reproduces this result, and give an explicit expression for the non-trivial (Wilson-Fisher) fixed point $V^{*}\left(\varphi^{2}\right)$ and for the perturbations about this fixed point. These solutions play an important rôle for us, because we obtain from them numerically, by relaxation ( $c f$. appendix A), the solutions at finite $N$.

Taking the $N=\infty$ limit of equations (34) - (37), we obtain,

$$
\begin{aligned}
\frac{\partial}{\partial t} V+(1+\eta) \varphi^{2} V^{\prime}-3 V & =-\frac{(1-\eta / 4)}{\sqrt{K}\left(2 V^{\prime}+2 \sqrt{K}\right)^{1 / 2}} \\
\frac{\partial}{\partial t} K+(1+\eta) \varphi^{2} K^{\prime}+\eta K & =-\frac{(1-\eta / 4) K^{\prime}}{\sqrt{K}\left(2 V^{\prime}+2 \sqrt{K}\right)^{3 / 2}} \\
\frac{\partial}{\partial t} Z+(1+\eta) \varphi^{2} Z^{\prime}+(1+2 \eta) Z & =\cdots
\end{aligned}
$$

The equations for $K$ and $V$ have decoupled from the equation for $Z$, and all the equations are now first order equations.

The exactly soluble large $N$ limit arises when the interactions in $\Gamma_{t}$ (or the bare action $S_{\Lambda_{0}}$ ) are functionals only of $\varphi^{2}$ [16]. Evidently, this is true of $V$ in (9), while all such derivative squared interactions can be cast (by integration by parts if necessary) in the form $\frac{1}{8}\left(\partial_{\mu} \varphi^{2}\right)^{2} Z\left(\varphi^{2}, t\right)$. This coincides with the $Z$ term in (9) but rules out a non-trivial $K$. Therefore we require $K\left(\varphi^{2}, t\right) \equiv 1$. This is indeed a solution of (50), but only if we also set $\eta=0$.

Note that if we take $\eta=0$, then from (50) the fixed point solution has to be trivial: $K\left(\varphi^{2}, t\right) \equiv 1$. It would be very interesting to perform a thorough search of the two parameter space (e.g. parametrized by $V(0)$ and $\eta$ ) of $t$-independent solutions to (49,50), looking for non-singular non-trivial solutions, which would if they exist, thus correspond to novel fixed points with non-trivial $K$ and $\eta \neq 0$. From the above analysis, such solutions for $K$ would lie outside the exactly soluble sector of the large $N$ limit [16].

We will see that non-trivial perturbations $\delta K$ around the Wilson-Fisher fixed point, which (from the above) also lie outside the exactly soluble sector, yield very sensible results for the eigen-value spectrum.

\footnotetext{
${ }^{3}$ We are free to choose the normalization to be unity by the scaling symmetry (11).
} 
Setting $\eta=0$, and $K\left(\varphi^{2}\right)=1$, we have that the fixed point potentials satisfy,

$$
\varphi^{2} V^{\prime}-3 V=-\frac{1}{\left(2 V^{\prime}+2\right)^{1 / 2}}
$$

Differentiating with respect to $\varphi^{2}$ and setting $W\left(\varphi^{2}\right)=V^{\prime}\left(\varphi^{2}\right)$ we then have,

$$
-2 W+\varphi^{2} W^{\prime}=\frac{W^{\prime}}{(2+2 W)^{3 / 2}}
$$

This is trivially solvable as a differential equation for $\varphi^{2}$ with respect to $W$,

$$
\varphi^{2}= \pm A \sqrt{W}+\frac{1}{2 \sqrt{2}} \sqrt{1+W}\left(1+\frac{W}{1+W}\right)
$$

where $A \geq 0$ is a constant of integration. $A>1 / \sqrt{2}$ corresponds to the line of Gaussian fixed points that appear at $N=\infty$ [15, 16], with the negative sign root for $\varphi^{2}<1 / 2 \sqrt{2}$, and the positive sign root for $\varphi^{2}>1 / 2 \sqrt{2}$. $A=1 / \sqrt{2}$ is the end-point where the Bardeen-Mosher-Bander phenomenon appears [31, 15]. $0<A<1 / \sqrt{2}$ is not allowed, because there exists a range of field values $\varphi^{2}<\varphi_{c}^{2}$ for which no real solution $W$ exists. The value $A=0$ is the only remaining possibility, and is the solution we take since this corresponds to the WilsonFisher fixed point [11, 15, 16]. Inverting (54) then gives two solutions, only one of which satisfies the original equation (53) however:

$$
W=\left(\varphi^{2}\right)^{2}-1 / 2+\varphi^{2} \sqrt{1+\left(\varphi^{2}\right)^{2}}
$$

Integrating this solution and comparing with (52), gives the potential:

$$
V=-\frac{1}{2} \varphi^{2}+\frac{1}{3}\left(\varphi^{2}\right)^{3}+\frac{1}{3}\left\{\left(\varphi^{2}\right)^{2}+1\right\}^{3 / 2} .
$$

Substituting $K\left(\varphi^{2}\right)=1$ and $\eta=0$ into (51), we find

$$
\begin{aligned}
& \varphi^{2} Z^{\prime}+Z=\frac{4-\eta}{\pi}\left\{-I_{2,0,2} Z^{\prime}+\frac{1024}{3} I_{4,3,2} V^{\prime \prime 4}\left(\varphi^{2}\right)^{2}\right. \\
& +\frac{32}{3} \varphi^{2}\left(4 I_{6,2,3}-8 I_{4,2,3}+I_{4,2,2}+4 I_{2,2,3}-5 I_{2,2,2}+I_{2,2,1}\right) V^{\prime \prime} V^{\prime \prime \prime} \\
& +\frac{64}{3} \varphi^{2}\left(16 I_{5,3,2} Z \varphi^{2}-8 I_{5,2,3}-11 I_{3,2,2}+8 I_{3,2,3}-8 I_{3,3,2}+8 I_{3,3,1}+8 I_{5,3,2}\right) V^{\prime \prime 3} \\
& +\left(\frac{256}{3} \varphi^{2} Z I_{6,3,2}-\frac{256}{3} \varphi^{2} Z I_{4,3,2}+\frac{256}{3} \varphi^{2} Z I_{4,2,3}-\frac{416}{3} \varphi^{2} Z I_{4,2,2}\right. \\
& +\frac{256}{3}\left(\varphi^{2}\right)^{2} Z^{2} I_{6,3,2}+\frac{128}{3} I_{6,2,3}-\frac{256}{3} I_{4,2,3}-64 I_{4,2,2}+\frac{128}{3} I_{2,2,3}-\frac{64}{3} I_{2,2,2}
\end{aligned}
$$




$$
\begin{aligned}
& -\frac{64}{3} I_{2,2,1}+\frac{128}{3} I_{6,0,5}+\frac{64}{3} I_{6,3,2}+\frac{128}{3} I_{4,3,1}-\frac{128}{3} I_{6,1,4}+\frac{256}{3} I_{4,1,4}+\frac{80}{3} I_{4,1,3} \\
& +\frac{128}{3} I_{2,0,5}+\frac{16}{3} I_{2,0,3}-32 I_{2,0,4}+\frac{64}{3} I_{2,3,0}+\frac{64}{3} I_{2,3,2}-\frac{128}{3} I_{2,1,4}+\frac{112}{3} I_{2,1,3} \\
& +\frac{16}{3} I_{2,1,2}-\frac{128}{3} I_{2,3,1}-\frac{32}{3} I_{4,0,4}-\frac{256}{3} I_{4,0,5}-\frac{128}{3} I_{4,3,2}+\frac{256}{3} \varphi^{2} Z I_{4,3,1} \\
& \left.-\frac{256}{3} \varphi^{2} Z I_{6,2,3}\right) V^{\prime \prime 2}+\left(\frac{8}{3} \varphi^{2} Z^{\prime} I_{3,2,1}+\frac{8}{3} Z I_{5,2,2}-\frac{40}{3} \varphi^{2} Z^{\prime} I_{3,2,2}-\frac{64}{3} \varphi^{2} Z^{\prime} I_{5,2,3}\right. \\
& -\frac{40}{3} Z I_{3,2,2}+\frac{8}{3} Z I_{3,2,1}+\frac{32}{3} Z I_{3,2,3}-\frac{64}{3} Z I_{5,2,3}+\frac{32}{3} \varphi^{2} Z^{\prime} I_{7,2,3}+8 Z I_{3,0,3} \\
& \left.\left.+\frac{32}{3} Z I_{7,2,3}+\frac{32}{3} \varphi^{2} Z^{\prime} I_{3,2,3}+\frac{8}{3} \varphi^{2} Z^{\prime} I_{5,2,2}\right) V^{\prime \prime}\right\},
\end{aligned}
$$

where the $I_{j, k, m}$ were defined in (36). Needless to say, the above repesents a great simplification compared to the full $Z$ equation [29]. An analytic solution of this equation might be possible, by the methods of ref.[16], but the numerical solution is straightforward. Imposing the constraint that $Z$ exists for all $\varphi^{2}$ we get the solution shown in figure 4 .

To calculate the critical exponents we again linearize about the fixed point by writing $V\left(\varphi^{2}, t\right)=V^{*}\left(\varphi^{2}\right)+\varepsilon \mathrm{e}^{\lambda t} v\left(\varphi^{2}\right)$ and $K\left(\varphi^{2}, t\right)=K^{*}\left(\varphi^{2}\right)+\varepsilon \mathrm{e}^{\lambda t} k\left(\varphi^{2}\right)$, where $V^{*}$ and $K^{*}$ denote the fixed point solutions found above. From (49) and (50),

$$
\begin{aligned}
\lambda v+\varphi^{2} v^{\prime}-3 v & =\frac{1}{2} \frac{k+2 v^{\prime}}{(2+2 W)^{3 / 2}}+\frac{1}{2} \frac{k}{\sqrt{2+2 W}} \\
\lambda k+\varphi^{2} k^{\prime} & =\frac{k^{\prime}}{(2+2 W)^{3 / 2}},
\end{aligned}
$$

where $W$ is given in (55). These equations are straightforwardly soluble: e.g. from (53) and (58) we have by inspection

$$
k=a_{k}(W / 2)^{-\lambda / 2},
$$

while substituting $v=k\left(\varphi^{2}\right) f\left(\varphi^{2}\right)$, one finds

$$
v\left(\varphi^{2}\right)=\tilde{a}_{v}\left(\frac{W}{2}\right)^{3 / 2-\lambda / 2}+\left\{\frac{4 W^{2}+2 W-1}{\sqrt{2+2 W}}\right\} \frac{k\left(\varphi^{2}\right)}{4} .
$$

(These expressions may be further simplified by defining $z=\mathrm{e}^{\sinh ^{-1} \varphi^{2}}$, then $W=z^{2} / 2-1$ and the multiplier of $k / 4$ above, becomes $z^{3}-3 z+1 / z$.)

Noting the simple zero of $W\left(\varphi^{2}\right)$ at $\varphi^{2}=1 / 2 \sqrt{2}$, we see that (59) and (60) are non-singular if and only if: either $a_{k}=k=0$ and $\lambda=3,1,-1, \cdots$, the exactly

\footnotetext{
${ }^{4}$ following an observation by Haruhiko Terao
} 
soluble potential perturbations and spectrum found previously [11, 8, 15, 16]; or $\tilde{a}_{v}=0$ and $\lambda=0,-2,-4, \cdots$. The $\lambda=0$ solution of this set is the redundant perturbation (47). The others lie outside the large $N$ exactly soluble sector (as discussed above) and are thus presumably only approximate, however we see that their spectrum can be understood, as with the potential perturbations, by assigning scaling dimension $\left[\varphi^{2}\right]=2$ to $\varphi^{2}$, regarding this as a composite field. This interpretation seems reasonable in view of the explicit appearance of such a field with this dimension in traditional large $N$ approaches [22].

\section{Exact Results at $N=-2$}

The fact that $N=-2$ is also an exactly solvable case was noted long ago [24, 25]. The initial hope was to be able to combine the known results at $N=\infty$ with those at $N=-2$, by using Padé approximants, to gain information about the physically interesting cases $N=0,1,2,3$ [24, 25, 26]. It was found that when $N=-2$, the exponents are always Gaussian, $\eta=0$ and $\nu=1 / 2$. We will show how the derivative expansion reproduces these results at the leading order of the approximation. At the end of the section, we also outline some interesting behaviour we observe at $N=-4$.

Differentiating (20) with respect to $\varphi^{2}$, and setting $\varphi^{2}=0$ we have that fixed point potentials $V\left(\varphi^{2}\right)$ satisfy,

$$
-2 V^{\prime}(0)=-\frac{N+2}{\left\{2+2 V^{\prime}(0)\right\}^{3 / 2}} V^{\prime \prime}(0)
$$

Thus at $N=-2, V^{\prime}(0)=0$. Substituting this into (20), we find $V(0)=-\sqrt{2} / 3$. Therefore in this case, $V(0)$ and $V^{\prime}(0)$ are exactly determined, but $V^{\prime \prime}(0)$ is an $a$ priori free parameter. Numerically, we find that $V^{\prime \prime}(0)$ is fixed by the requirement that $V\left(\varphi^{2}\right)$ be non-singular for all $\varphi^{2} \geq 0$. We find two solutions: the trivial tricritical Gaussian fixed point $V\left(\varphi^{2}\right)=-\sqrt{2} / 3$, and a non-trivial Wilson-Fisher fixed point with $V^{\prime \prime}(0)>0$, satisfying the required asymptotic conditions at large $\varphi^{2}$ (cf. equation (22)).

Now consider the eigenperturbations. Differentiating (24) with respect to $\varphi^{2}$, and setting $\varphi^{2}=0$ we have

$$
(\lambda-2) v^{\prime}(0)=\frac{(N+2) v^{\prime \prime}(0)}{\left\{2 V^{\prime}(0)+2\right\}^{3 / 2}}-3 \frac{(N+2) v^{\prime}(0) V^{\prime \prime}(0)}{\left\{2 V^{\prime}(0)+2\right\}^{5 / 2}} .
$$

Hence when $N=-2, \lambda=2$ or $v^{\prime}(0)=0$. For the case $\lambda=2$, we checked numerically that there exists a non-singular solution with the required asymptotic 
behaviour (26). This eigenvalue yields $\nu=1 / 2$. For the case $v^{\prime}(0)=0$, setting $\varphi^{2}=0$ in (24) we find $(\lambda-3) v(0)=0$. Thus either $\lambda=3$, which corresponds to the trivial vacuum energy perturbation $v\left(\varphi^{2}\right) \equiv 1$, or $v(0)=0$.

In the latter case we now have $v(0)=v^{\prime}(0)=0$, so for non-trivial solutions of this type we may normalise $v^{\prime \prime}(0)=1$. We expect from Sturm-Liouville theory [5] that about each fixed point, for an infinite discrete set of eigenvalues $\lambda<2$, these solutions have the required asymptotic behaviour (26). . The perturbations about the Gaussian fixed point can be determined exactly: it is easy to check from (24), that the eigenperturbations with the required asymptotic behaviour, correspond to the Laguerre polynomials [14, 15] $v\left(\varphi^{2}\right)=2 L_{n}^{N / 2-1}\left(\varphi^{2} \sqrt{2}\right)$ (given by the formula $L_{n}^{\alpha}(y)=\frac{1}{n !} \mathrm{e}^{y} y^{-\alpha} \frac{d^{n}}{d y^{n}}\left(\mathrm{e}^{-y} y^{n+\alpha}\right)$ [32]) with $\lambda=3-n, n=0,1, \cdots$. For $n \geq 2$, these indeed satisfy $v(0)=v^{\prime}(0)=0$ and $v^{\prime \prime}(0)=1$.

As pointed out in Fisher 25] we should also expect to find exact solutions for $N=-4,-6, \cdots$. For $N=-4$ we find, in a similar way to above, that $V^{\prime \prime \prime}(0)$ is now an a priori free parameter, while either $V^{\prime \prime}(0)=V^{\prime}(0)=0$ and $V(0)=-2^{3 / 2} / 3$, or $V^{\prime \prime}(0)=12^{3 / 2} / 5^{5 / 2}, V^{\prime}(0)=1 / 5$ and $V(0)=-\sqrt{20 / 27}$. We have not checked however, that non-singular solutions actually exist with these boundary conditions (apart from of course the Gaussian solution $V^{\prime \prime \prime}(0)=0$ allowed by the first set). The first set of boundary conditions correspond to Fisher's findings [25], since we then obtain $(\lambda-2)(\lambda-1) v^{\prime}(0)=0$, so that the Gaussian-type eigenvalues $\lambda=1,2$ are recovered when acceptable solutions exist with $v^{\prime}(0) \neq 0$. (Additionally it may be shown that $v^{\prime \prime}(0)=0$ if $\lambda \neq 1$ or 2.) The second set leads to $\left(6 \lambda^{2}-9 \lambda-10\right) v^{\prime}(0)=0$, suggesting the existence of a non-trivial fixed point with two exactly soluble eigenvalues $\lambda=3 / 4 \pm \sqrt{321} / 12$.

To confirm this finding (at this level of approximation) would require however to check that a non-singular fixed point solution actually exists, as mentioned above, and that eigenperturbations satisfying (26) for large $\varphi^{2}$, exist with the above eigenvalues. Equally, we expect to recover Fisher's results at $N=-6,-8, \cdots$, and further novel candidates for fixed points. We do not pursue these interesting questions further.

\section{$7 \quad$ Numerical Results}

In the table, we display the numbers only to the accuracy necessary for comparison to the worlds best estimates. We did not find second order results for $N=0$,

\footnotetext{
'This is not at variance with 25] since the source term $J \cdot \varphi$ in (11) generates 'anisotropic' 25] correlation functions.
} 


\begin{tabular}{|c||c|c||c|c|c||c|c|c|}
\hline \multicolumn{1}{|c||}{$N$} & \multicolumn{2}{c||}{$\eta$} & \multicolumn{3}{c||}{$\nu$} & \multicolumn{3}{c|}{$\omega$} \\
\hline & $O\left(\partial^{2}\right)$ & World & $O\left(\partial^{0}\right)$ & $O\left(\partial^{2}\right)$ & World & $O\left(\partial^{0}\right)$ & $O\left(\partial^{2}\right)$ & World \\
\hline 0 & & $.030(3)$ & .596 & & $.590(2)$ & .62 & & $.81(4)$ \\
\hline 1 & .054 & $.035(3)$ & .66 & .618 & $.631(2)$ & .63 & .897 & $.80(4)$ \\
\hline 2 & .044 & $.037(4)$ & .73 & .65 & $.671(5)$ & .66 & .38 & $.79(4)$ \\
\hline 3 & .035 & $.037(4)$ & .78 & .745 & $.707(5)$ & .71 & .33 & $.78(3)$ \\
\hline 4 & .022 & $.025(4)$ & .824 & .816 & $.75(1)$ & .75 & .42 & \\
\hline \hline 10 & .0054 & .025 & .94 & .95 & .88 & .89 & .82 & .78 \\
\hline 20 & .0021 & .013 & .96 & .98 & .94 & .95 & .93 & .89 \\
\hline 100 & .00034 & .003 & .994 & .998 & .989 & .991 & .988 & .98 \\
\hline
\end{tabular}

Table 1: Critical exponents of the three-dimensional Wilson-Fisher fixed point. The first two orders of the derivative expansion are compared to a combination of the worlds best estimates [22, 35], with their errors, where available. $O\left(\partial^{0}\right)$ is the leading order in the derivative expansion, where $\eta$ is identically zero for all $N$, and $O\left(\partial^{2}\right)$ is the second order in derivative expansion.

for numerical reasons, as explained in appendix A.

Since, as we have seen, already the Local Potential Approximation yields the known exact results at $N=-2,-4, \cdots$, and at $N=-\infty$, at the very least these approximations provide physically motivated interpolations between these exact values, which thus go beyond the early hopes of using Padé approximants [24, 25, 26].

In fact there is quite an impressive agreement between $O\left(\partial^{0}\right)$ and estimates by other methods. Compared to these other methods, at first there is a gradual decrease in the accuracy of the prediction for $\nu$, as $N$ increases, whilst the prediction for $\omega$ shows a slight improvement, until as $N \rightarrow \infty$ all exponents become exactly determined. The results compare favourably with those found by other authors, both using a different form of cutoff [6, 15, 21, 33, 34] and those obtained using a sharp cutoff [3, 0, 8, 12. Ref. 15 displays a table comparing results from different forms of the Local Potential Approximation.

At $O\left(\partial^{2}\right)$, for $N=1 \cdots 4$, the results are already rather accurate for $\eta$ and $\nu$, showing an improvement on the leading order results. This is also true of $\omega$ at $N=1$, but for $N=2,3,4$ the accuracy of $\omega$ is poorer than at $\left(\partial^{0}\right)$. For the 
large $N$ cases however, $\omega$ is well estimated, $\nu$ is not improved at $O\left(\partial^{2}\right)$, while $\eta$ is dramatically underestimated - eventually by about a factor of 10 .

With the results exact at $N=\infty$, we see that there is a loss of accuracy in the approach to this limit. Indeed the approximation scheme appears 'too biased' towards the $N=\infty$ results. As we speculated in ref. 四, this may be because all the appropriate fields have not been included in the effective action, in this regime. Indeed, it is known that at large $N$, a massless bound state field also exists at the critical point 22, 35]. We should thus expect that a derivative expansion is not so well behaved, because the vertex functions are hiding within them the effects of this integrated out massless field. To ameliorate this behaviour, we should include the bound state explicitly as an effective $O(N)$ singlet field, then amongst the new set of fixed points in this enlarged space will be one with the same universal properties as the original $N$ vector model, but with better behaved derivative expansion properties. We expect that similar considerations will apply to fixed points with fermions, particularly since the bound state fields here also correspond to the order parameter (a.k.a. fermion condensate) [36].

\section{Acknowledgements}

TRM would like to thank Geoff Golner for useful comments on $N=-2$, and PPARC for support through an advanced fellowship, and grant GR/K55738. MDT would like to thank PPARC for support through a studentship.

\section{A Numerical Methods}

In this section we outline the methods used to solve numerically our equations. We describe in particular, how to cast the equations manifestly as coupled second order differential equations, how the asymptotic expressions were used to set boundary conditions, and why it is necessary to develop these beyond leading order for $K$ and $V$, and yet one further order for $Z$. We explain why relaxation was our method of choice for these problems, and why analytic forms for the integrals $I_{i, j, k}$ proved insufficient. We describe the singular behaviour seen as $N \rightarrow 1$, and the relaxation from the exact results at $N=\infty$ that was used

instead. We also point out some of the (simpler) issues that arose on solving for the perturbations.

All the equations that we needed to solve numerically, form two point bound- 
ary value problems. There are two methods for solving such equations: shooting [37, 38] and relaxation [38. Both methods were used, however relaxation is particularly suited to equations and boundary conditions involving complicated expressions which cannot be solved in closed form: there is no need to write the equation explicitly in the form $d y / d x=f(x, y)$. It is also the best method when one needs to find solutions that depend upon some parameter, such as the value of $N$. Once a solution for one value of $N$ is found, one can use this solution as an initial guess for a close value of $N$. Given the nature of our problem it is not surprising that relaxation turned out to be our principal method.

We formulate the equations as a set of non-linear, coupled second order differential equations, as follows [19]. Noting that the equation for $Z, c f$. (37), [29], or for $N=\infty$ (57), contains powers of $V^{\prime \prime \prime}$, we differentiate the $V$ equation (34) and thus find an expression for $V^{\prime \prime \prime}$ in terms of $V, V^{\prime}, V^{\prime \prime}, K, K^{\prime}, Z$ and $Z^{\prime}$. This is substituted back into the $Z$ equation. Similarly, the $\varphi^{2}=0$ boundary condition for the $Z$ equation, contains powers of $V^{\prime \prime}(0)$. We eliminated these in favour of $V(0), V^{\prime}(0), K(0)$ and $Z(0)$ by using the differentiated $V$ equation. Similar transformations are required to turn the equations for the perturbations into manifestly second order differential equations.

For the other set of boundary conditions we choose a value of $\varphi^{2}$, which we call $\varphi_{\text {asy }}^{2}$, large enough such that the asymptotic expressions in appendix B become a good enough approximation to the solutions [19, 20]. (These expressions go beyond leading order. The leading order expressions $(38)-(40)$ are not sufficient, as explained below.) We then use the asymptotic expressions for $V, K$ and $Z$ to provide boundary conditions for $V, V^{\prime}, K, K^{\prime}, Z$ and $Z^{\prime}$. The value of $\varphi_{\text {asy }}^{2}$ must not be chosen so large that numerical instability prevents us from obtaining a solution. There is a certain amount of trial and error in deciding where to set $\varphi_{\text {asy }}^{2}$ : we cannot really decide where to set it until we know something about the solution. We checked that the solutions were stable against reasonable changes in the value of $\varphi_{\text {asy }}^{2}$.

It is necessary to develop the asymptotic expressions to beyond the leading order in order to find a solution [19]. Thus for example in (20), if we just substitute the leading order results, viz. the first term in (22), for $V\left(\varphi_{\text {asy }}^{2}\right)$ and $V^{\prime}\left(\varphi_{\text {asy }}^{2}\right)$, and attempt to solve (20) for $V^{\prime \prime}\left(\varphi_{\text {asy }}^{2}\right)$ (as in effect takes place in the numerical procedures), we see that there is no real solution for any finite $\varphi_{\text {asy }}^{2}$. Hence, we are forced to expand the asymptotic expressions to beyond leading order. A similar result holds for $K\left(\varphi^{2}\right)$. However, when we consider $Z\left(\varphi^{2}\right)$, we see that we need to take the asymptotic expressions to next-to-next-to-leading order: the correction to the leading order asymptotic behaviour does not involve $Z^{\prime \prime}$, hence the above procedure carried only to next-to-leading order leaves $Z^{\prime \prime}$ incorrectly determined. 
In order to ensure that $Z^{\prime \prime}$ gets determined correctly (at least to leading order), we need to extend the asymptotic expression yet one further order.

In the perturbed equations it is sufficient to use the leading order asymptotic expression [19, 20]. As a check we calculated the corrections to the leading order behaviour and also used these. This made no difference to the values quoted.

The solution of the equations is complicated by the fact that they are stiff [37, 38. This arises because small perturbations from the true solutions lead to a singular behaviour. Relaxation is particularly adept at handling stiff problems. In this way, the numerical solution of the leading order equations proved to be easy. It was only necessary to provide an initial guess and the relaxation routines quickly found the solution.

The integrals (36) play an important part in the solution of the second order equations. It is possible to find a general analytic result for them, but these turned out not to be useful for the present purposes. The integrals are generally small numbers, but in the analytic expressions these small numbers often arise from the cancellation of large numbers. In this case roundoff errors play too large a part in determining their value using the analytic expression. Even performing the calculations using higher precision Fortran routines could not provide accuracy beyond four significant figures in extreme (but realised) cases. Furthermore, whilst the equations (34) - (37) contain about three hundred terms expressed in terms of the integrals $I_{i, j, k}$, particularly for the larger $i, j, k$ the algebraic expressions are so large that the equations expressed in closed form stretch to between twenty five and thirty thousand terms. The main effect of this dramatic increase is cumulative roundoff error. The attained level of accuracy proved totally insufficient and so numerical methods were used. We used an adaptive integrator from the NAG libraries. The problem was split into two parts: a first part from zero up to some finite value $q_{s}$, and the remaining bit. To avoid roundoff errors we used an expression arising from asymptotic analysis to calculate the second part of the integral, and only used the numerical routines to compute the integral over the finite range first part. The point $q_{s}$ was set to be as small as possible (to avoid numeric round off in the numerical integration), whilst still allowing the asymptotic expression to be accurate.

The numerical solution of the second order equation is then made difficult by the large number of integrals that need to be calculated numerically during each iteration. It was soon noted that the computational power required could not be provided by normal workstations. Parallel Fortran code, making use of MPI [39], was developed to run on a sixteen node IBM SP2 system. This reduced run times for the relaxation code from up to one week to typically between ten minutes to one hour. 
Initially we tried to solve the second order equations by relaxing away from $N=1$ using the numerical solutions obtained in [19] as initial guesses. However, this procedure failed because as $N \rightarrow 1$ the fixed point solution $Z\left(\varphi^{2}\right)$ becomes rapidly sharper at the origin, diverging as $Z\left(\varphi^{2}\right) \sim 1 / \varphi^{2}$ in the limit $N=1$. This was confirmed by substituting $N=1$ and $K=\kappa-Z \varphi^{2}$ [cf. (48)] in the $K$ equation (35), using the known results [19 for $\kappa$ and $V$, and solving for $Z$. This divergence is allowed because at $N=1$ only the combination $\kappa=K+Z \varphi^{2}$ has any meaning and this remains well behaved, as explained at the end of section 4. Our successful strategy was to first solve the simpler $N=\infty$ equations, and then relax from $1 / N=0$ to finite $N$ using these solutions as initial guesses.

The $N=\infty, V$ and $K$ equations were solved analytically as described in section 5. The equation (51) that determines $Z$ at $N=\infty$, was solved numerically. Being first order, there is only one boundary condition, which is that the solution exists for all $\varphi^{2}$ and thus has the required asymptotic behaviour. For this problem we used the shooting method. An initial value of $Z$ was used to integrate the equation out towards the second boundary using an eight point Runge-Kutta integrator. In general the initial value $Z(0)$ would be wrong: if it was too large then the solution would tend to infinity at some finite $\varphi^{2}$; if it was too small the solution would tend to minus infinity at some finite value of $\varphi^{2}$. The correct value was then found by binary chop. It was particularly difficult to get past the point $\varphi^{2}=1 / 2 \sqrt{2}$. This is because at this point the right hand side of the equations has a $0 / 0$ type behaviour, i.e. $Z^{\prime}=n\left(\varphi^{2}, Z\right) / d\left(\varphi^{2}, Z\right)$, such that as $\varphi^{2} \rightarrow 1 / 2 / \sqrt{2}, n\left(\varphi^{2}, Z\right) \rightarrow 0$ and $d\left(\varphi^{2}, Z\right) \rightarrow 0$, for the true solution. There are general methods of dealing with such singular points [38], but our equations were sufficiently well behaved to avoid needing to use them.

The shooting method was tried for $1 / N>0$ also, but this proved to be unsuitable. This is because to use shooting effectively we need to be able to reach the other boundary or have a particularly simple set of equations (e.g. the $N=\infty$ equations). The singularity structure of the equations makes it impossible to shoot from one side to the other and hence makes the more complicated problem unsuited to shooting.

The main difficultly in solving the second order equation was removing the instabilities in $Z$ near the origin. These manifested themselves in the form of sharp spikes in $Z^{\prime}$ near $\varphi^{2}=0$. These were removed by writing $Z\left(\varphi^{2}\right)=s\left(\varphi^{2}\right) \tilde{Z}\left(\varphi^{2}\right)$, where $s\left(\varphi^{2}\right)$ is a known scaling function that makes $\tilde{Z}\left(\varphi^{2}\right)$ as flat as possible. This greatly speeded up the calculations and removed the spikes in the solutions. However even this could not quell the instabilities that arise as $N \rightarrow 1$ where $Z(0)$ actually diverges (as discussed above). As a consequence we found that accurate solutions even at $N=2$ were much harder to obtain than those of $N=3$ (say), 
while the singular behaviour at $N=1$ prevented us from relaxing to $N=0$. This is the reason why we do not display $N=0$ results at second order in table 1 .

In the equations for the perturbations we calculated the parts dependent only upon the fixed point solutions and stored them in a file, thus saving a large computational overhead [19, 20]. It was important that these functions, which are the functions that multiplied $v, v^{\prime}, v^{\prime \prime}, k$ etc. in each of the linearized equations, were well determined. The size of the $z$ equation was such that small errors in the fixed point solutions made it difficult to determine these functions accurately, for large $\varphi^{2}$. This problem was solved by determining these functions using the asymptotic expressions for $V, K$ and $Z$ (cf. appendix B) and then matching them onto the ones calculated from the fixed point solutions.

To conclude, we note that we checked that the eigenvalues were stable against

reasonable changes in where $\varphi_{\text {asy }}^{2}$ was set, in the number of mesh points, and other numerical factors. A good indicator of numerical accuracy was to find numerically the redundant perturbation mentioned in section 4 , and check that it matched the analytic form and that its eigenvalue vanished to good precision. (These properties are only completely recovered in the limit of an infinitely fine mesh.) We found we could numerically determine this perturbation and eigenvalue to a high degree of accuracy for all values of $N$.

\section{B Asymptotic expressions for $V, K$ and $Z$}

From the second order equations (34) - (37), we determined the asymptotic behaviour of $V\left(\varphi^{2}\right)$ and $K\left(\varphi^{2}\right)$ for large $\varphi^{2}$ to next-to-leading order, and $Z\left(\varphi^{2}\right)$ to next-to-next-to-leading order, for reasons explained in appendix A. With $A_{v}, A_{k}$ and $A_{z}$ denoting constants, the results are

$$
\begin{aligned}
& V\left(\varphi^{2}\right) \sim A_{v}\left(\varphi^{2}\right)^{\left(3 \frac{1}{1+\eta}\right)}+ \\
& \quad\left(\frac{1}{24} \frac{\sqrt{6}(N-1) \sqrt{1+\eta}}{\sqrt{A_{v}} \sqrt{A_{k}} N}+\frac{1}{24} \frac{\sqrt{6}(1+\eta)}{\sqrt{A_{v}} N \sqrt{A_{k}+A_{z}} \sqrt{5-\eta}}\right)\left(\varphi^{2}\right)^{\left(\frac{-1+\eta}{1+\eta}\right)}
\end{aligned}
$$




$$
\begin{aligned}
& K\left(\varphi^{2}\right) \sim A_{k}\left(\varphi^{2}\right)^{\left(-\frac{\eta}{1+\eta}\right)}+2\left(\frac { 1 } { 8 6 4 } \sqrt { 6 } ( 1 + \eta ) \left(4 \eta^{4} A_{k}{ }^{3}+16 A_{k}{ }^{2} \eta^{4} A_{z}\right.\right. \\
& +11 A_{k} \eta^{4} A_{z}{ }^{2}+48 \eta^{3} A_{z}{ }^{3}+55 A_{k} \eta^{3} A_{z}{ }^{2}-52 A_{k}{ }^{3} \eta^{3} \\
& -56 A_{k}{ }^{2} \eta^{3} A_{z}-579 \eta^{2} A_{k} A_{z}{ }^{2}-480 A_{k}{ }^{2} \eta^{2} A_{z}-80 A_{k}{ }^{3} \eta^{2} \\
& -144 \eta^{2} A_{z}{ }^{3}-703 \eta A_{k} A_{z}{ }^{2}+336 \eta A_{k}{ }^{3}+40 A_{k}{ }^{2} \eta A_{z} \\
& \left.-432 \eta A_{z}{ }^{3}-80 A_{k} A_{z}{ }^{2}-240 A_{z}{ }^{3}+160 A_{k}{ }^{2} A_{z}\right) /\left(\sqrt{A_{k}+A_{z}}\right. \\
& \left.\left(-4 A_{k}+2 A_{k} \eta+A_{z}+\eta A_{z}\right)^{2}(5-\eta)^{3 / 2} N A_{v}{ }^{3 / 2}\right)+\frac{1}{864} \sqrt{6} \\
& \sqrt{1+\eta}\left(\left(12 \eta^{3} N+28 \eta^{3}+48 \eta N-48 \eta^{2} N-32 \eta^{2}-48 \eta\right) A_{k}{ }^{3}\right. \\
& +\left(68 \eta^{2} A_{z}-24 \eta N A_{z}-12 \eta^{2} N A_{z}+44 \eta^{3} A_{z}+8 \eta A_{z}\right. \\
& \left.+12 \eta^{3} N A_{z}-16 A_{z}\right) A_{k}{ }^{2}+\left(3 \eta^{3} N A_{z}{ }^{2}+3 \eta N A_{z}{ }^{2}+21 \eta^{3} A_{z}{ }^{2}\right. \\
& \left.+69 \eta A_{z}{ }^{2}+66 \eta^{2} A_{z}{ }^{2}+24 A_{z}{ }^{2}+6 \eta^{2} N A_{z}{ }^{2}\right) A_{k}+5 \eta^{3} A_{z}{ }^{3} \\
& \left.+15 \eta A_{z}{ }^{3}+15 \eta^{2} A_{z}{ }^{3}+5 A_{z}{ }^{3}\right) /\left(A_{v}{ }^{3 / 2} \sqrt{A_{k}} N\right. \\
& \left.\left.\left((2 \eta-4) A_{k}+\eta A_{z}+A_{z}\right)^{2}\right)\right)\left(\varphi^{2}\right)^{\left(-\frac{4}{1+\eta}\right)} \\
& Z\left(\varphi^{2}\right) \sim A_{z}\left(\varphi^{2}\right)^{\left(-\frac{1+2 \eta}{1+\eta}\right)}+\left(\frac { 1 } { 1 4 4 } \left(64 \eta^{5} N-65 \eta^{5}+185 \eta^{4}-172 \eta^{4} N-716 \eta^{3} N\right.\right. \\
& +652 \eta^{3}+604 \eta^{2} N-452 \eta^{2}-2524 \eta+2348 \eta N-1184 \\
& +1264 N) \sqrt{6} /\left(\sqrt{A_{v}} N(-2+\eta)^{2}(1+\eta)(5-\eta)^{3 / 2}\right) \\
& \left.+\frac{1}{24} \frac{\sqrt{6}\left(20 \eta^{4}-33 \eta^{3}-6 \eta^{2}-58 \eta-24\right)(N-1)}{(1+\eta)^{3 / 2}(-2+\eta)^{2} N \sqrt{A_{v}}}\right)\left(\varphi^{2}\right)^{\left(-3 / 2 \frac{\eta+2}{1+\eta}\right)}
\end{aligned}
$$




\section{References}

[1] K. Wilson and J. Kogut, Phys. Rep. 12C, 75 (1974).

[2] T. Morris, Int. J. Mod. Phys. A9, 2411 (1994).

[3] T. Morris, Phys. Lett. B334, 355 (1994).

[4] T. Morris, in RG96, SHEP 96-25, hep-th/9610012.

[5] T. Morris, SHEP 96-36, hep-th/9612117, to be published in Nucl. Phys. B.

[6] N. Tetradis and C. Wetterich, Nucl. Phys. B422, 541 (1994).

[7] M. Alford, Phys. Lett. B336, 237 (1994).

[8] K-I. Aoki et al, Prog. Theor. Phys. 95, 409 (1996).

[9] J. Nicoll, T. Chang and H. Stanley, Phys. Rev. Lett. 33, 540 (1974).

[10] T. Morris, Nucl. Phys. B458, 477 (1996).

[11] F. Wegner and A. Houghton, Phys. Rev. A8, 401 (1973).

[12] A. Hasenfratz and P. Hasenfratz, Nucl. Phys. B270, 685 (1986).

[13] T. Morris, Phys. Lett. B357, 225 (1995).

[14] T. Morris, Phys. Rev. Lett. 77, 1658 (1996).

[15] J. Comellas and A. Travesset, UB-ECM-PF 96/21, hep-th/9701028.

[16] M. D'Attanasio and T. Morris, SHEP 97-03, hep-th/9704094.

[17] G. Golner, Phys. Rev. B8, 339 (1973);

A. Filippov and A. Radievsky, Phys. Lett. A169, 195 (1992).

[18] G. Golner, Phys. Rev. B33, 7863 (1986).

[19] T. Morris, Phys. Lett. B329, 241 (1994).

[20] T. Morris, Phys. Lett. B345, 139 (1995).

[21] R. Ball et al, Phys. Lett. B347, 80 (1995).

[22] J. Zinn-Justin, Quantum Field Theory and Critical Phenomena, Clarendon Press, Oxford, 1989. 
[23] R. Pisarski and F. Wilczek, Phys. Rev. D29, 338 (1984);

Y. Iwasaki et al, Phys. Rev. Lett. 78, 179 (1997).

[24] R. Balian and G. Toulouse, Phys. Rev. Lett. 30, 544 (1973).

[25] M. Fisher, Phys. Rev. Lett. 30, 679 (1973).

[26] M. Fisher, Rev. Mod. Phys , 597 (1974).

[27] A. Margaritis, G. Ódor and A. Patkós, Z. Phys. C39 109, (1988).

[28] J. Vermaseren, KEK-preprint-92-1, 1992.

[29] The $Z$ flow equation is available as pub/9706/Z.tex, by anonymous ftp from hep2.phys.soton.ac.uk

[30] F. Wegner, J. Phys. C7, 2098 (1974).

[31] W.A. Bardeen, M. Moshe and M. Bander, Phys. Rev. Lett. 52 (1984) 1188; F. David, D.A. Kessler and H. Neuberger, Phys. Rev. Lett. 53 (1984) 2071, Nucl. Phys. B257 (1985) 695.

[32] See e.g. I.S. Gradshteyn and I.M. Ryzhik, Table of integrals, series, and products, Academic Press.

[33] J. Adams et al., Mod. Phys. Lett. A10, 2367 (1995).

[34] J. Berges, N. Tetradis, and C. Wetterich, Phys. Rev. Lett. 77, 873 (1996).

[35] Y. Okabe and M. Oku, Prog. Theor. Phys. 60, 1277 (1978);

K. Kanaya and S. Kaya, Phys. Rev. D51, 2404 (1995);

S. Ma, Phys. Rev A10, 1818 (1974).

[36] Such theories have been considered by J. Comellas, Y. Kubyshin and E. Moreno, hep-th/9512086, hep-th/9601112; Y. Kubyshin, in RG96, hepth/9702082.

[37] J. Stoer and R. Bulirsch, Introduction to Numerical Analysis, Springer, 2nd edition edition, 1993.

[38] W. Press, S. Teukolsky, W. Vetterling, and B. Flannery, Numerical Recipes: the art of scientific computing, Cambridge University Press, 1986.

[39] M. P. I. Forum, Mpi: A message passing interface standard, University of Tennesse, Report No. CS-94-230, 1994, See also International Journal of Supercomputing Applications volume 8, number 3/4, 1994 and http://www.mcs.anl.gov/mpi/mpi-report/mpi-report.html. 